\title{
Primary pleural squamous cell carcinoma accompanied by a big pulmonary bulla in a 68 -year- old man: a case report and literature review
}

Chuan Zhong ( $\sim$ zchuanhappy@126.com )

Suining Central Hospital https://orcid.org/0000-0001-9852-3632

Haining Zhou

Suining Central Hospital

Ramón Rami-Porta

Hospital Universitari MutuaTerrassa

Yunfei Zhao

Suining Central Hospital

\section{Case report}

Keywords: Primary pleural squamous cell carcinoma, Surgical resection, Radiotherapy, Pulmonary bulla

Posted Date: July 8th, 2020

DOI: https://doi.org/10.21203/rs.3.rs-40421/v1

License: (c) This work is licensed under a Creative Commons Attribution 4.0 International License.

Read Full License 


\section{Abstract}

Background: Primary pleural squamous cell carcinoma is extremely rare. Its incidence is not described in the literature, and the cause of this disease is unclear due to its rarity.

Case presentation: We present the rare case of primary pleural squamous cell carcinoma in a 68-year-old man with a big pulmonary bulla who undergone en bloc complete resection of both lesions. Two months after surgery, the patient received radiation therapy over the operative field to a total dose of 50Gy. More than a year after completion of treatment, the patient is well and in complete remission.

Conclusions: To the best of our knowledge, this is the first case of primary pleural squamous cell carcinoma with a big pulmonary bulla. Complete surgical resection combined with radiation therapy was proposed for therapeutic purposes. To

investigate long-term prognosis of this disease, further more cases are needed.

\section{Background}

Epithelial neoplasms arising from the pleura are almost exclusively represented by mesothelial tumors such as malignant mesothelioma, but other histotypes do exist [1]. Primary pleural squamous cell carcinoma (PPSCC) is exceedingly rare [2]. Due to the extreme rarity of PPSCC, its incidence is not described in the literature, and the cause of this disease is unclear $[3,4]$. We report the rare case of a patient with a PPSCC associated with a big pulmonary bulla who underwent surgical resection.

\section{Case Presentation}

A 68-year-old man presented with a 4-month history of right-sided chest pain as the only complaint. The patient had no history of echinococcosis, tumor or tuberculosis; however, he had been smoking 20 cigarettes and drinking 100 grams of alcohol a day for 40 years. Computed tomography(CT) revealed a right anterolateral pleural thickening, a soft tissue mass in the adjacent chest wall, a big bulla measured $4.4 \times 5.1 \mathrm{~cm}$ in the lung, and normal mediastinal lymph nodes (Fig. 1A-C). No positive lesions was found after a series of workup, including cranial magnetic resonance imaging(MRI), contrast-enhanced neck and abdomianl CT scans, bronchoscopy and radionuclide bone scintigraphy. The tentative diagnosis was localized mesothelioma with pulmonary bulla. Right thoracotomy via the third intercostal space was performed. Intraoperative exploration found that the mass in the subcutaneous tissue measured $4 \times 5 \mathrm{~cm}$, and was continuous with a pleural lesion measuring $5 \times 6 \mathrm{~cm}$, which had poorly demarcated margins and had invaded the big bulla of the lung. Intraoperative frozen biopsy of the mass revealed malignant tumor, which tended to be squamous cell carcinoma. En bloc resection of the tumor and the bulla was performed, including the chest wall, pleura, part of the third and fourth ribs, tissues of the intercostal space, and the big pulmonary bulla by wedge resection. The appropriate type of nickeltitanium memory alloy embracing device was fixed to the third and fourth rib stumps to avoid local collapse of the chest wall. Postoperative histopathological report revealed squamous cell carcinoma invading the adjacent ribs 
and pulmonary bulla with chronic inflammation (Fig. 2A-D). Morphological diagnosis was completed with immunohistochemical staining: P63 and P40 were positive (Fig. 2E-F); TTF1, Napsin A, WT1, D2-40, c-kit and Calretinin were negative. Given the lack of radiological evidence of tumor involvement of the lung, mediastinum, head, neck and abdomen, the disease was clinically considered primary to the pleura. Postoperative course was uneventful. Two months after surgery, the patient returned to our hospital to undergo radiation therapy over the operative field to a total dose of $50 \mathrm{~Gy}$. After more than 1 year's followup, there are no signs of recurrence (Fig. 1D-F).

\section{Discussion}

PPSCC is extremely rare and it has been described in very few case reports. Pathogenesis of PPSCC is actually unknown, because of its extreme rarity [4]. Some authors suggest that chronic pleural inflammation could be one of the causes of PPSCC [1]. The participation of chronic inflammation in the pathogenesis of this tumor is also suggested by the fact that some of the reported cases occurred in patients with empyema cavity with pleurocutaneous fistula [5]; with extrapleural pneumothorax, without fistula, for treatment of tuberculosis [5]; with tuberculosis [6]; and with bronchopleural fistula due to bronchiectasis [7]. In our case, the presence of a big pulmonary bulla with chronic inflammation in the vicinity of the tumor may have promoted neoplastic changes in the adjacent pleura with the final result of squamous cell carcinoma. There are no specific symptoms in PPSCC and the tumor is usually detected on CT or Positron Emission Tomography-Computed Tomography(PET-CT). Preoperative biopsy, especially transthoracic Tru-Cut needle biopsy, is instrumental for the diagnosis of pleural tumors, but the reported rate of accurate diagnosis is not satisfactory [3]. The differential diagnosis of the present case included localized mesothelioma, primary or secondary chest wall tumors, pleural metastasis, and thymic carcinoma. Pleura-based tumor spread was indeed reminiscent of mesothelioma; however, diffuse expression of p40 and p63 and negative expression of WT1, Calretinin and D2-40 ruled out this possibility $[4,8,9]$. Primary chest wall tumors arise from muscle, fat, blood vessel, nerve sheath, cartilage, or bone of the chest wall; nevertheless, postoperative histopathological report revealed squamous cell carcinoma, which also precluded that possibility. Due to no history of tumor and the lack of radiological evidence of tumor involvement of the lung, mediastinum, head, neck and abdomen, secondary chest wall tumors and pleural metastasis were excluded. Although most thymic carcinoma takes the form of squamous cell carcinoma, the current tumor did not involve the mediastinum and lacked expression of ckit, a highly sensitive marker for thymic carcinoma [8].

Surgical resection is the treatment of choice for localized tumors [4]. The two patients reported by Rüttner et al. were surgically treated and were free of disease 3 and 5 years after the operation [5]. The patient described by Prabhakar et al. died from massive hemorrhage five month after resection, and local or distance recurrence could not be ruled out because necropsy was not performed [7]. A patient with extensive PPSCC was treated with nivolumab after six cycles of cisplatinum and docetaxel and local radiation therapy. Although there was some tumor response, the tumor progressed locally and distantly and the patient died two year after diagnosis [10]. Finally, Yoshida et al. reported on a 33-year-old female with no history of inflammatory disease or smoking who presented with an extensive PPSCC that had a 
deficiency of SMARCB1 (INI1), a tumor-suppressor gene, the loss which has been associated with malignant tumors of the kidney, gastrointestinal tract, pancreas and uterus. Although intensively treated with chemotherapy, the patient died 10 months after diagnosis [8]. The evidence is scarce, but radiotherapy after complete resection may improve the prognosis of this disease, and this was the rationale followed with our patient.

\section{Conclusion}

We encountered the first case of a PPSCC with a big pulmonary bulla in an old man. Complete surgical resection combined with radiation therapy was associated with mid-term disease-free survival. Long-term prognosis of this disease are uncertain at present.

\section{Abbreviations}

PPSCC: Primary pleural squamous cell carcinoma; CT: Computed tomography; MRI: cranial magnetic resonance imaging; PET-CT: Positron Emission Tomography-Comp- uted Tomography

\section{Declarations}

\section{Acknowledgements}

None.

\section{Authors' contributions}

$\mathrm{CZ}$ and $\mathrm{HZ}$ wrote the manuscript. $\mathrm{CZ}$ and $\mathrm{HZ}$ performed the surgery. $\mathrm{YZ}$ carried out the pathological examinations. RRP and $C Z$ were involved in the final editing. All authors read and approved the final manuscript.

\section{Funding}

None.

\section{Availability of data and materials}

Not applicable.

\section{Ethics approval and consent to participate}

This present study was approved by the Ethics Committee of Suining Central Hospital.

\section{Consent for publication}


Written informed consent was obtained from the patient for publication of this case report and any accompanying images.

\section{Competing interests}

The authors declare that they have no competing interests.

\section{References}

1. Galateau-Salle F, Churg A, Roggli V. The World Health Organization committee for Tumors of the Pleura. The World Health Organization.

2. Franke $M$, Chung HD, Johnson FE. Squamous cell carcinoma arising from the pleura after pneumonectomy for squamous cell carcinoma of the lung. Am J Surg. 2010;199:e34-5.

3. Lin XM. Chi C, Chen J. Liu Y, Li P. Yang Y. Primary pleural squamous cell carcinoma misdiagnosed as localized mesothelioma: a case report and review of the literature. J Cardiothorac Surg 2013; 8:50.

4. Ronchi A, Cozzolino I, Montella M. Primary pleural squamous cell carcinoma: A diagnostic challenge. Cytopathology. 2018;29:205-7., Vicidomini G, Morgillo F, Della Corte C M, et al.

5. Rüttner JR, Heinzl S. Squamous-cell carcinoma of the pleura. Thorax. 1977;32:497-500.

6. Garty I, Straussberg R, Flatau E. Extraosseous 99mTc-MDP uptake in squamous cell carcinoma of the pleura. Eur J Nucl Med. 1987;12:589 - 91.

7. Prabhakar G. Mitchell IM, Guha T. Norton R. Squamous cell carcinoma of the pleura following bronchopleural fistula. Thorax 1989;44:1053-4.

8. Yoshida K, Fujiwara Y, Goto Y. The first case of SMARCB1 (INI1)-deficient squamous cell carcinoma of the pleura: a case report. BMC Cancer. 2018;18:398. https://doi.org/10.1186/s12885-018-4321-X., Kohno T, Yoshida A, Tsuta K, et al. .

9. Ordóñez NG. Application of immunohistochemistry in the diagnosis of epithelioid mesothelioma: a review and update. Human Pathology, 44(1), 1-19. doi:10.1016/j. humpath.2012.05.014.

10. Sigala I. Alevizopoulos N, Elefteriou K. Gianniou N, Kalomenidis I. Primary squamous cell carcinoma of the pleura treated with nivolumab. Respirology Case Reports 2020;8:e00516. doi:

$10.1002 /$ rcr2.516.

\section{Figures}




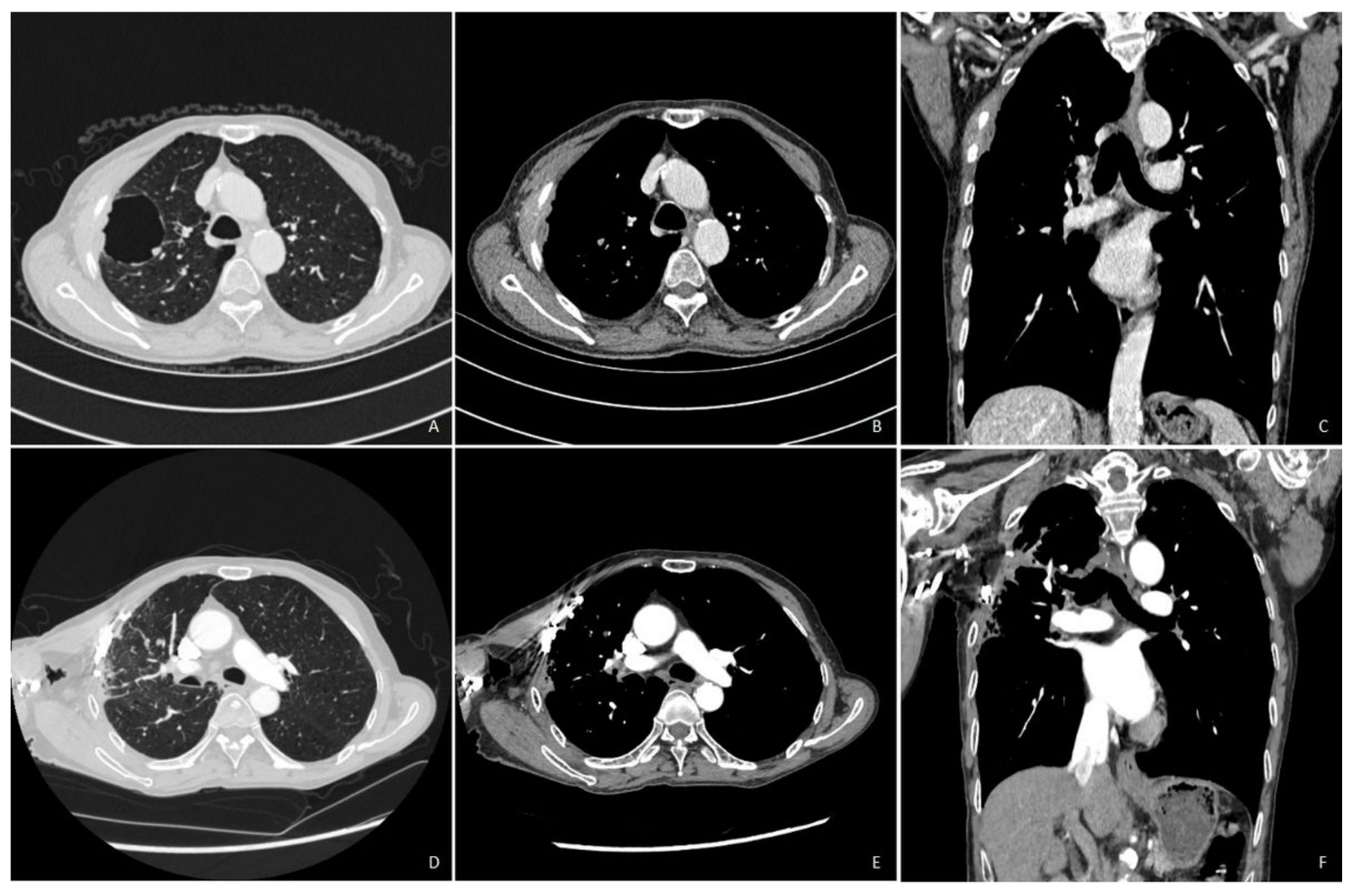

Figure 1

Chest CT scan before operation (A-C); chest CT scan more than 1 year's follow-up after surgery (D-F).

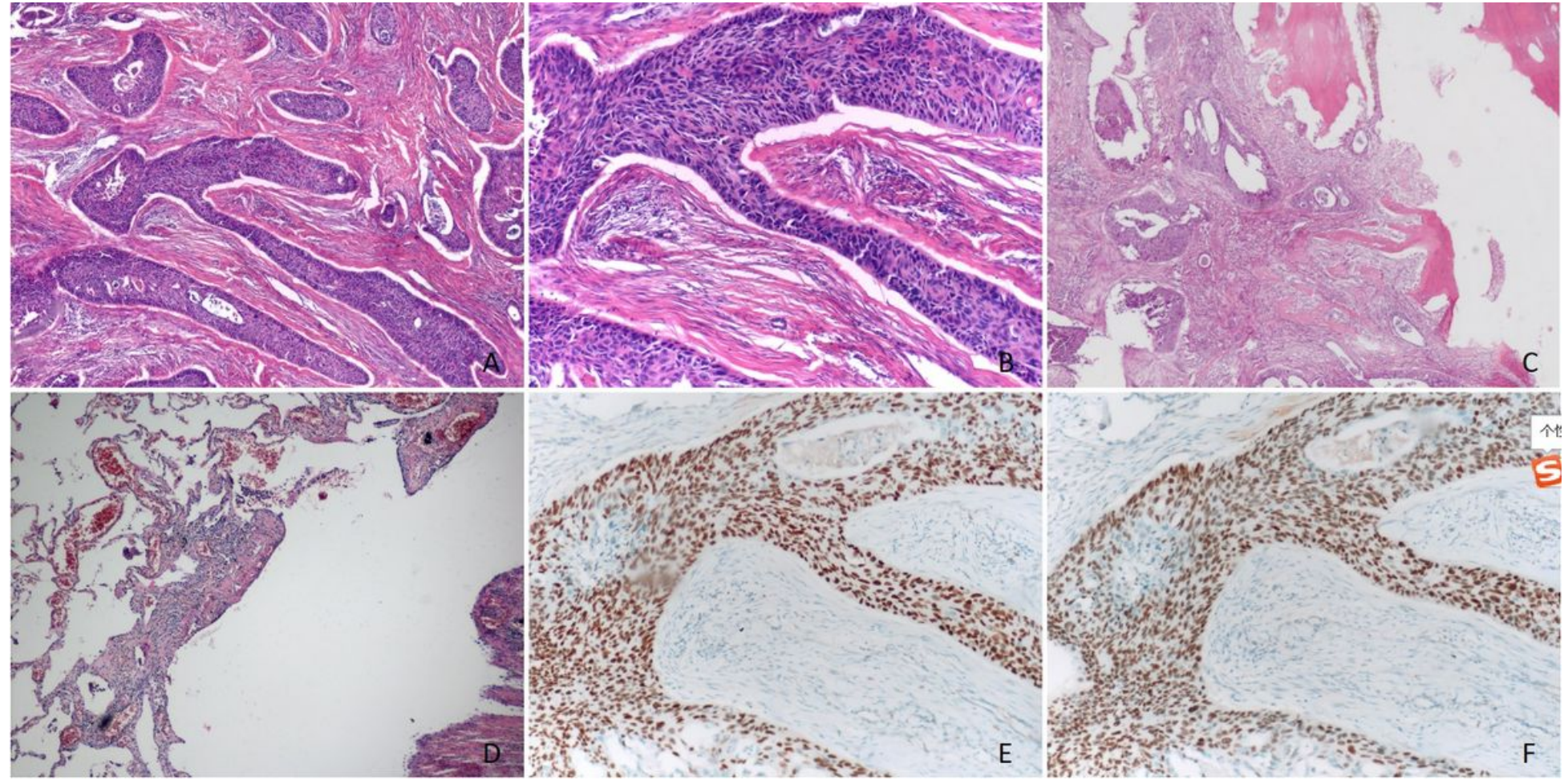




\section{Figure 2}

(A) The neoplastic cells are arranged in the form of nests with different sizes and have distinct intercellular bridges(H\&E stain, magnification x40). (B) The cells were round or ovoid, with abundant cytoplasm, large nuclei, obvious nucleoli, and intracellular keratinization in focal areas (H\&E stain, magnification $\times 100$ ). (C) Squamous cell carcinoma invading the adjacent ribs (H\&E stain, magnification $x 40$ ). (D) Pulmonary bulla with chronic inflammation (H\&E stain, magnification x40). (E-F) Neoplastic cells immunohistochemically stained with the use of antisera against p40 and p63, respectively. Neoplastic cells show clear nuclear positivity for both (E) p40 and (F) p63. 